\title{
Carcinoma inflamatorio de la mama
}

\author{
Ana Luiza Da Cruz Ríos de Sánchez*; Fernando de Miranda Henriques**; José Antonio Ribeiro Filho***
}

RESUMEN: Los autores presentan su experiencia en la evaluación y tratamiento del Carcinoma Inflamatorio de la Mama (CIM) en la Unidad de Mastología del Hospital de Base del Distrito Federal-Brasilia, durante el período de 1977 a 1988. Los aspectos: Histórico, Clínico, Estudios Paraclínicos, Diagnóstico Diferencial, Anatomía Patológica, Tratamiento y Pronóstico son ampliamente revisados a la luz de su propia experiencia y de los datos obtenidos de la literatura médica.

La necesidad de pensar en el CIM en los casos de Mastitis atípica para poder iniciar el tratamiento adecuado a la mayor brevedad posible, es enfatizada bien como la tendencia mundial contraria a la mastectomía como tratamiento inicial del CIM.

PALABRAS CLAVES: Carcinoma Inflamatorio de la mama - Tratamiento.

SUMMARY: The authors present their experience in the evaluation and treatment of the Inflammatory Carcinoma of the Breast (ICB) in the Mastology Unit of the Hospital de Base do D.F. Brasilia, during the period of 1977 through 1988. The Historical, Clinical, Para-clinical Studies, Diferencial Diagnosis, pathological Anatomy, treatment and Prognosis are widely studied on the light of their own experience and of the information obtained from the Medical Literature.

The idea of begginning the fastest as possible when appearance of Atypical Mastitis a treatment against ICB is emphazised and also the world tendency against the Mastectomy as the initial treatment of the Inflamatory Carcinoma of the Breast.

KEY WORDS: Inflamtory Carcinoma of the Breast - Treatment.

\section{Introducción}

Charles Bell en 1814 fue el primero en establecer que "cuando una coloración rojiza y puntadas de dolor acompañan el tumor de la mama significaun mal comienzo".(1) Volkmann, en 1985, denominó esta entidad de "mastitis carcinomatosa". (2) Lee y Tannebaum (1924) introdujeron el término de CARCINOMA INFLAMATORIO utilizado hasta nuestros días (3).

El concepto clínico de Carcinoma Inflamatorio de la Mama, fue establecido por Haagensen: "carcinoma inflamatorio consiste en la presencia de un tumor mamario acompañado de eritema y edema de la piel de la mama, en por lo menos un tercio de su superficie". (4) Ellis y Tetelbaun (1974) establecieron la invasión vascular y de los linfáticos dérmicos como condición "sine qua no" para el diagnóstico de carcinoma inflamatorio (5).

Donnely (1948) dividió el Carcinoma Inflamatorio de la Mama (CIM) en dos tipos:

Primario: las señales inflamatorias surgen simultáneamente con el carcinoma en la piel, en una mama previamente normal.

Secundario: manifestaciones inflamatorias que surgen en la mama que presenta cáncer esquirroso, o en la otra mama,

\footnotetext{
* Ginecóloga. Pasantía de Mastología en Hospital de Base. Brasilia D.E. - Brasil

** Ginecólogo. Presidente de la Sociedad Brasileña de Mastología.

*** Ginecólogo. Presidente de la Sociedad Brasileña de Mastología.
}

pudiendo también surgir después de la mastectomía en el sitio original o en la mama opuesta (6).

En 1967, José Baptista de Silva Neto, estableció como CIM secundario aquellos cuyas señales inflamatorias surgen acompañando una reincidencia tumoral en la cicatriz de la mastectomía siempre que el eritema y el edema sean también amplios y NO HAYA INFECCION (7).

Lucas y Pérez-Mesa definieron como CARCINOMA INFLAMATORIO OCULTO la invasión carcinomatosa de los linfáticos dérmicos sin señales clínicas de inflamación (89). En este mismo trabajo retrospectivo estos autores estableciron tres grupos de pacientes: el primero presentaba únicamente señales clínicas, el segundo clínicas y patológicas y el tercero solamente un criterio histológico. La evolución clínica, la diseminación y la rápida evolución fatal fue prácticamente igual en los tres grupos aunque ligeramente mejor para el diagnóstico clínico como pilar terapéutico y de pronóstico para la paciente.

En el presente estudio revisamos las historias de 11 (once) pacientes con diagnóstico de carcinoma inflamatorio de mama diagnosticados entre 1977 y enero de 1988, en la UNIDAD DE MASTOLOGIA DEL HOSPITAL DE BASE DEL DISTRITO FEDERAL, comparando nuestros datos con los obtenidos por otros autores.

\section{Incidencia}

El CIM constituyó entre el 1\% hasta el 9,3\% de los casos de carcinoma mamario según las diferentes series $(4,6,7)$. La mayoría de los autores cree que representa entre 1,7 a 2,5 de 
todos los carcinomas de mama. (Haagensen, 1971; Anderson 1980; Chu y col., 1980).

En nuestra serie la paciente de más edad tenía 65 años y la más joven 34 , lo que concuerda con la literatura. Cinco pacientes premenopáusicas y 6 posmenopáusicas.

En el grupo de nuestras pacientes únicamente una se encontraba gestante. Se trataba de una paciente de 34 años, G2 P1 que estaba en el cuarto mes de embarazo cuando llegó a consulta por inflamación y dolor en la mama izquierda de 2 meses de evolución. En el examen se encontró convexidad de la mama izquierda, con eritema de 80 a $90 \%$ de su superficie, "peau d'orange" y edema del pezón. Es difícil la delimitación del tumor por ser muy doloroso el tacto. No había signos clínicos de compromiso extramamario, el diagnóstico de carcinoma inflamatorio fue clínico y confirmado por citopunción y "forage" (drill-biopsy). La baja incidencia de carcinoma inflamatorio en gestantes en nuestra serie está de acuerdo con los datos de muchos autores $(6,7,12-14)$ siendo la incidencia semejante a la de otras formas de cáncer mamario.

\section{Diagnóstico clínico}

Robbins y col. (11) resaltó los principales signos de la enfermedad: 1 . Eritema con margen erisipeloide; 2 . Aumento de la temperatura de la piel que recubre el tumor y la mama afectada; 3 . Endurecimiento de los tejidos mamarios subyacentes. La retracción del pezón y el fenómeno de (piel de naranja) también pueden ocurrir (3). Se observa la ulceración de la piel en cerca de un quinto de los casos (11).

En nuestra serie, la incidencia de la enfermedad fue la misma tanto en la mama derecha (6 casos) como en la izquierda por tacto. Unicamente una paciente presentó una masa menor de $5 \mathrm{~cm}$. En la serie de Chu y col. (1980) citada por Oliveira y Urbanetz (6), el tamaño promedio de la lesión fue de $6,2 \mathrm{~cm}$ y en 36 de las 62 pacientes estudiadas (58\%), los tumores fueron mayores de $5 \mathrm{~cm}$. El eritema y el edema estuvieron presentes en todas las pacientes. Dos pacientes presentaban fijación de tumor a la piel, dos tenían trayectos fistulosos con drenaje de material purulento, "peau d'orange" en cuatro casos, desviación del pezón en cuatro casos y tumor fijo a los planos profundos en dos casos. Una paciente presentaba además del tumor principal lesiones nodulares satélites.

Haagensen (1971), considerando que la progresión de las alteraciones inflamatorias es generalmente rápida y que el dolor acompaña la enfermedad en gran número de pacientes, calculó que el intervalo entre la observación del primer síntoma y la búsqueda de atención médica sería de dos meses y medio en promedio.

En el grupo estudiado, el tiempo más corto de búsqueda de ayuda médica fue de 5 meses y el más largo de una paciente de 36 años con historia de nódulo doloroso en la mama izquierda de 4 años de evolución. Esta paciente llegó en una etapa avanzada de la enfermedad presentando también "peau d'orange" y compromiso ganglionario. Este hecho de la demora de buscar ayuda médica está de acuerdo con el bajo nivel económico e intelectual de nuestras pacientes y del poco acceso a las instituciones hospitalarias.

La mayoría de nuestras pacientes acusó la aparición de una masa dolorosa como motivo principal de consulta. Unicamente dos de las pacientes referían apenas eritema y endureciemiento y una de ellas sólo buscó al médico después de que comenzó a drenar material purulento. Esto está en desacuerdo con el relato de varios autores (9-11) provenientes de países donde el nivel de sus pacientes permite detectar y tratar la enfermedad en etapas más tempranas.

En cuatro pacientes había compromiso ganglionario homolateral; en una, compromiso axilar heterolateral, una con ganglios supraclaviculares y en tres, hepatomegalia.

\section{Estudio paraclínico}

El estudio hematológico de las pacientes portadoras de carcinoma inflamatorio no muestra leucocitosis ni anormalidades características excepto una discreta elevación de la velocidad de sedimentación globular y anemia secundaria. $(4,12-13)$. Según Haagensen, la presencia de leucocitosis coincide con metástasis para el tejido blando, pulmón, hueso o cerebro (4).

\section{Mamografía y termografía}

La mayoría de los autores está de acuerdo con que el CIM es una entidad pobre en señales radiológicas. $(4,6,12-14)$. Las señales radiológicas más frecuentes y más sugestivas de carcinoma inflamatorio son: edema intenso de la piel, tanto en lo que se refiere al espesor de la dermis como a su extensión, siempre mayor que dos tercios de la superficie cutánea (14); aumento por infiltración de trama glandular con prominencia de los vasos linfáticos subcutáneos $(6,14)$.

Unicamente a tres de nuestras pacientes les fue solicitada mamografía siendo que una presentó la señal de Leborgne (nódulo palpable mayor que el nódulo radiológico) e invasión de los linfáticos de la piel; en otra fue reportado un nódulo de $1,2 \mathrm{~cm}$ sugestivo de ser maligno (bordes irregulares, edema de la piel) y la tercera mamografía no identificó nódulo característico o calcificaciones reportando únicamente una gran área densa, no homogénea, en los cuadrantes externos de predominio superior compatible con lesión inflamatoria sin descartar neoplasia asociada.

La termografia en pacientes con CIM muestra aumento difuso de temperatura de la mama comprometida en relación con la mama normal (13). Torre y col., citado por Oliveira Urbanetz (6), establece criterios diagnósticos característicos de CIM: 1)Zonas hipertérmicas de gran intensidad (gradiente siempre superior a $4-5^{\circ} \mathrm{C}$ ); 2) extensión de la hipertermia tumoral a la totalidad de la glándula frecuentemente sobrepasando los límites de la misma; 3) marcada hipervascularización, muy hipertérmica, de morfología anárquica, especialmente visible en la periferia de las áreas hipertérmicas, en muchas ocasiones con abundante drenaje para la cadena de la mamaria interna.

Collier y Pinotti (14) hacen referencia a un patrón vascular TIPO V4 -Estrella Vascular- compatible con carcinoma Inflamatorio.

Tan sólo una de nuestras pacientes fue sometida a la Termografía que fue reportada como placas de calor difuso, anarquía vascular; pezón caliente y señal de edema de piel. El examen fue catalogado como Clase $\mathrm{D}$, o sea, caso termográficamente maligno de pronóstico malo.

\section{Citopunción y drenaje (drill-biopsy)}

Desde el año de 1986 hace parte de la rutina diagnóstica de nuestro Servicio practicar a toda paciente con sospecha 
clînica de malignidad citopunción de la lesión sin aspiración y "drill-biopsy" ("forage"). Tres de nuestras pacientes tuvieron su diagnóstico confirmado por medio de estos métodos con positividad para tumor en todos los casos. Sin embargo, por las características intrínsecas de los métodos $(15,16)$ no es posible la confirmación histopatológica de la invasión por células tumorales. En la última paciente de nuestra serie, una gestante de 34 años, además del Forage se tomó un pequeño fragmento de piel para exámenes anatomopatológicos el cual no mostró invasión tumoral de los linfáticos dérmicos ni vascular, a pesar del cuadro clínico florido.

\section{Estudios inmunológicos}

Zylberberg y col. (17) estudiaron 15 pacientes con diagnóstico clínico de CIM. Como parte integrante del estudio dosificaron al antígeno Carcinoembrionario estudiado anteriormente en neoplasias mamarias o en otros tumores $(14,19)$. Cinco pacienteś del estudio tenían niveles de 10 a $24 \mathrm{ng}$ los cuales volvieron a la normalidad cuando iniciaron la quimioterapia. Mientras tanto tres pacientes que fallecieron nunca tuvieron, ni inicialmente, ni cuando las metástasis se hicieron evidentes, niveles del antígeno superiores a $8 \mathrm{ng}$.

En cuanto al "status", los autores notaron anergia inicial únicamente en tres casos (BCG y Croton negativos), las cuales recuperaron su capacidad de respuesta después de dos ciclos de quimioterapia (17).

\section{Receptores hormonales}

Delarue y col. dosificaron en 1981 receptores para estrógeno y progesterona en 59 pacientes con CIM y encontraron que el $47 \%$ fueron positivos para estrógeno y $34 \%$ para progesterona. Estos datos no difieren de la presencia de receptores en el total de los cánceres mamarios $(6,14,19,20)$.

\section{Diagnóstico diferencial}

Haagensen (4) enumeró las principales entidades clínicas que deben ser consideradas cuando una paciente con un cuadro sugestivo de CIM. Recientemente Oliveira y Urbanetz (1987) y Balogh (1983) revisaron este tópico. Entre los diagnósticos diferenciales se destacan:

1) Procesos infecciosos (mastitis infecciosa, abcesos) las cuales usualmente cursan con fiebre y muestran leucocitosis en el hemograma. Algunos autores están de acuerdo con una prueba terapéutica con tratamiento con antibióticos mientras se espera el resultado de la cultura y antibiograma de material de la lesión. En los casos de carcinoma inflamatorio esta cultura generalmente es negativa (12). Esta conducta se justifica principalmente en pacientes jóvenes, especialmente dentro del ciclo grávido-puerperal donde sus características especiales la vuelven vulnerable a las patologías infecciosas. Además, considerando el sombrío pronóstico de esta enfermedad no es conveniente hacer afirmaciones antes de estar plenamente seguro del diagnóstico.

2) En nuestro medio es importante destacar el papel de la sífilis y de la tuberculosis en el diagnóstico diferencial de estas pacientes.

3) Ectasia ductal, con o sin celulitis, generalmente se presenta como un proceso más localizado, con compromiso de menos de un tercio de la mama y con rápida respuesta a la terapia conservadora.
4) Erisipela, se caracteriza por la mama demasiado roja $\mathrm{y}$ con cuadro de dolor muy intenso, o que normalmente no ocurre en el CIM.

5) Neoplasias de la mama que se vuelven voluminosas y necróticas, en muchas ocasiones con infección secundaria y/ o crecimiento muy cercano a la piel.

6) Patologías más raras como el linfoma de la mama, sarcoma, cloroma (infiltración leucémica mielogénica) o invasión linfomatosa de la mama.

\section{Patología}

La biopsia es indispensable para establecer el diagnóstico de cáncer y ella fue positiva en todas nuestras pacientes. El CIM no está asociado a un patrón histológico específico (17, 18). Entretanto, formas indiferenciadas y de alto grado de malignidad son más frecuentes en esta modalidad de presentación tumoral $(17,18)$.

El trabajo pionero de Ellis y Teitelbaum en 1974 (5) definiendo histológicamente el CIM como la presencia de émbolos de células tumorales en los linfáticos dérmicos motivó una serie de estudios determinando la validez de cada modalidad diagnóstica y su aplicación en términos de conducta y pronóstico para la paciente. En este mismo año, Saltzstein (9) publicó cuatro casos que presentaban invasión tumoral de los linfáticos dérmicos sin sospecha clínica de CIM. En esta ocasión él propuso el término de Carcinoma Inflamatorio de la Mama Clínicamente Oculto. El curso clínico de esas pacientes fue compatible con el mal pronóstico de las pacientes con CIM diagnosticado clínicamente y el autor hizo la advertencia de la contraindicación quirúrgica en estos casos. Posteriormente Lucas y Pérez-Mesa (8) en una serie de 58 pacientes con CIM clínico y 15 con cáncer inflamatorio oculto concluyó que tanto el criterio clínico como el histológico eran válidos por lo menos para pronóstico a corto plazo.

En nuestros 11 casos, en una paciente ocurrió un carcinoma papilar invasor y en las otras 10 restantes carcinoma ductal infiltrante. La invasión vascular estuvo presente en cuatro casos. La invasión linfática fue mínima en 1 paciente y linfáticos dérmicos comprometidos en dos casos.

\section{Tratamiento y pronóstico}

En 1814 cuando Charles Bell hizo la primera descripción del carcinoma inflamatorio de la mama ya llamó la atención sobre el grave pronóstico (7). Haagensen (1971) se refirió al carcinoma inflamatorio de mama como la más terrible forma de cáncer de mama. (4) Gros y Hashnel, citados por Stocks y Patterson, establecieron que más del $50 \%$ de las pacientes portadoras de CIM mueren en 1 año independientemente del tipo de tratamiento instaurado (13).

En 1967, Silva Neto y Batista Vieira, publicaron su experiencia en el tratamiento de 51 casos de carcinoma inflamatorio. El promedio de sobrevida total de las 47 pacientes con seguimiento fue de 16,5 meses. En este trabajo realizado en el Instituto Central de la Asociación Paulista de Lucha contra el Cáncer durante el período de 1954-1961, los autores afirman su posición a favor de la MASTECTOMIA RADICAL siempre asociada a la irradiación en el pre o post operatorio. El argumento fue que se trataba del método que les había proporcionado los tres casos de sobrevida asintomática a 5 años y también los mejores índices de sobrevida total (7). 
Robbins y col. publicaron una gran serie de 186 casos de CIM atendidas en el Memorial Hospital de Nueva York durante el período de 1949 a 1971. De este grupo 83 pacientes eran portadoras de CIM. En este estudio solo fue utilizada la radioterapia si la enfermedad estaba clínicamente limitada a la mama y a la axila. La terapia hormonal y/o quimioterapia complementaron la radioterapia en algunas pacientes y promovieron en algunas ocasiones respuestas objetivas y corto plazo. No obtuvieron buenos resultados con oforectomía, excepto en una paciente. Entre 1950 y 1953, cuatro pacientes fueron sometidas a mastectomía radicales. Todas murieron en el lapso de un año posterior a la cirugía. En este artículo, los autores consideran que el carcinoma inflamatorio de la mama es una contraindicación absoluta para la práctica inicial de mastectomía o inclusive después de la radioterapia (11).

En 1976Ernest Camp 111 al reportar cuatro casos y revisar la literatura existente, llegó a la conclusión de que "la mastectomía radical representa un intento de radicalizar la cura de una enfermedad incurable" (21).

Ann M. Chu (1980), expresa claramente su opinión sobre la terapia en el CIM: "El consenso, con pocas excepciones, es que la cirugía radical es inútil y que los mejores resultados paliativos son obtenidos con radioterapia de alto voltaje". En su artículo ella y sus col. aplicaron 6000 rads en ochos semanas a la mama y pared toráxica a través de campos tangenciales opuestos con equipo de megavoltaje. De las 62 pacientes de la serie con CIM no metastática, entre enero de 1966 y diciembre de 1977, 50 fallecieron por la enfermedad, 7 se encontraban vivas en el momento de la publicación del artículo pero con la enfermedad y cinco se encontraban libres de la enfermedad. El promedio de sobrevida fue de 18 meses y $14 \%$ alcanzaron 5 años. El catorce por ciento tuvieron un período libre de la enfermedad de 5 años. Chu y col. postulan también la radioterapia en dosis fraccionadas dos veces diarias como una alternativa terapéutica en grandes tumores (mayores de $10 \mathrm{~cm}$ ) siendo que en su experiencia, hubo una disminución del 33\% de reincidencias locales (22).

El grupo de Buzdar, Montague, Barker, Hortobhyi y Blumenschein trató a 32 pacientes portadoras de CIM inicialmente con quimioterapia (Fluoracil, doxorubicina y ciclofosfamida) seguida de radioterapia comparando el intervalo libre de la enfermedad con un grupo de otras 32 pacientes tratadas anteriormente en su institución únicamente con radioterapia. Los autores concluyen que la modalidad de tratamiento combinado aumenta el intervalo libre de enfermedad en las pacientes menores de 50 años de edad, pero la tasa global de supervivencia no aumentó debido a la alta incidencia de compromiso de SNC. Entretanto, en las pacientes con 50 o más años de vida, el intervalo libre de la enfermedad aumentó como también la tasa global de supervivencia en $45 \%$ (23).

En 1982, Zylberberg y col. presentaron un estudio no randomizado de 15 pacientes con CIM en el cual intentaron mostrar las ventajas de la quimioinmunoterapia orientada por la termografía como arma terapéutica. El protocolo fue una combinación de Adriamicina, Vincristina, 5-fluoracil y metrotexate en aplicaciones, intravenosas y Melphalan vioral; aumentando de 1-BCG-Fen escarificación de la piel abdominal los dỉas 17 y 24 del ciclo de quimioterapia. El criterio de operabilidad era dado por el enfriamiento del tumor detectado por la Termografía, lo que generalmente ocurrió después del tercer ciclo de quimioterapia. El promedio de sobrevida de la serie excedía 56 meses en el momento de la publicación del artículo. Los autores atribuyen sus excelentes resultados a que la terapéutica loco-regional era iniciada únicamente después de la remisión de la enfermedad por la quimioterapia y diagnosticada por la Termografía de Placa (17).

Chacón, 1984, revisa la literatura y sugiere un esquema terapéutico combinado de hormonoterapia ablativa o aditiva, de acuerdo con la dosificación de receptores hormonales; quimioterapia y radioterapia. Recalca el estudio detallado de la extensión clínica (RX de tórax, fosfatasis alcalina, hidroxiprolina, cintilografía ósea y hepática, etc.) y la determinación de receptores hormonales (20).

Una gran serie de 170 casos de carcinoma inflamatorio de mama tratados con quimioterapia son comparados con 60 casos anteriores tratados con radioterapia y terapia hormonal. (24) Los resultados sugirieron que la quimioterapia inicial es muy útil en el tratamiento de este tipo de cáncer.

En nuestro servicio las conductas terapéuticas han variado de acuerdo con los estudios realizados en el país y fuera de el, de las ventajas y desventajas de los esquemas propuestos. En el grupo de nuestras pacientes realizamos mastectomía radical con salpingooforectomía en 1978 y, en 1980, la paciente volvió a buscar la Unidad con enfermedad sistémica con metástasis hepática y pulmonar falleciendo poco tiempo después. Una paciente fue tratada con mastectomía simple con disección de ganglios axilares, ooforectomía y radioterapia en 1979. En 1982, presentó múltiples metástasis óseas (cuello del fémur, calota craniana) y falleció. Tres pacientes fueron tratadas con mastectomía simple (higiénica) seguida de radioterapia. Una falleció dos años después y las otras dos

Tabla 1.

MANEJO

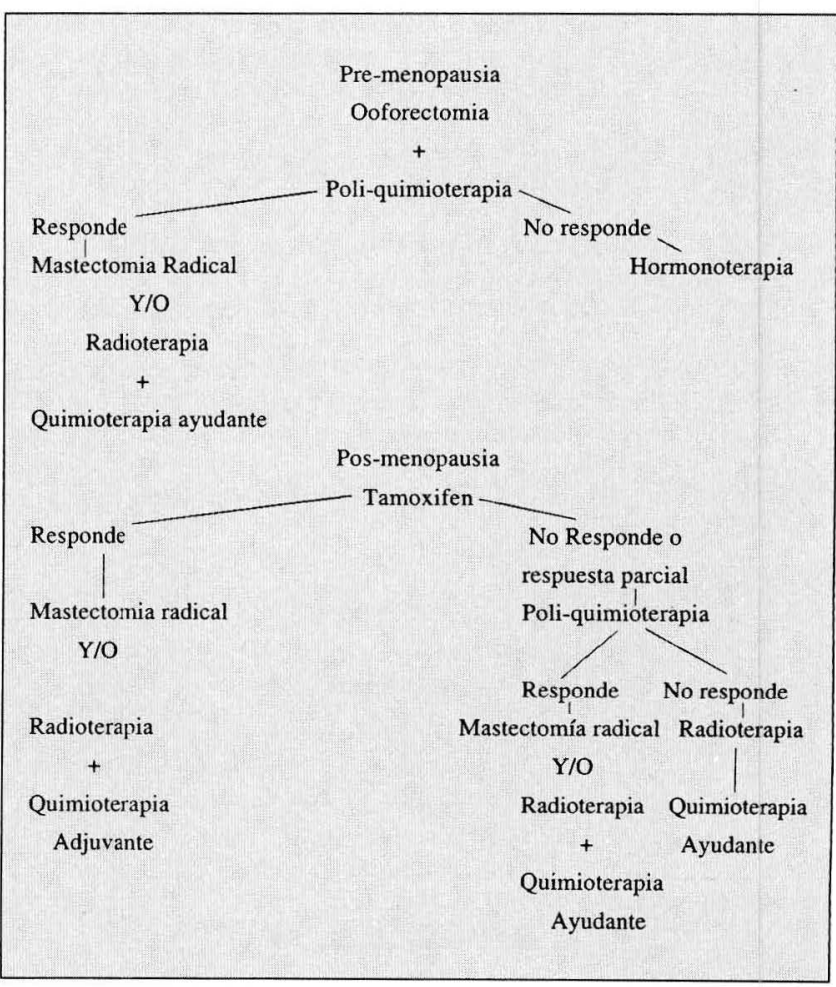


Tabla II

ESQUEMA TERAPEUTICO

\section{QUIMIOTERAPIA \\ 5 - Fluoracil \\ (500 $\mathrm{mg} / \mathrm{m}^{2}$ S.C.) \\ Adriamicina \\ (500 mg / de S.C.) \\ Ciclofosfamida $\left(500 \mathrm{mg} / \mathrm{m}^{2} \mathrm{de}\right.$ S.C. $)$}

Aplicación endovenosa cada 21 a 28 días de acuerdo con la tolerancia de la paciente.

RADIOTERAPIA

Mama o plastrón

Axila

Fosa Supraclavicar

$: 5.000$ Rads

:5.000 Rads

Mamaria Interna

$: 5.000$ Rads

Aplicación diaria de 200 Rads 5 días por semana.

no fue posible hacer el seguimiento. Cinco pacientes a partir de 1982 fueron incluidas en el esquema terapéutico de las tablas I yII que combina tratamiento hormonal, quimioterapia, radioterapia y mastectomía en casos seleccionados. De este grupo una paciente falleció 4 años después de iniciado el tratamiento. Las otras 4 pacientes permanecen vivas hasta el momento siendo la más antigua tratada desde 1983. La paciente gestante de nuestra serie, considerando la edad gestacional del momento del diagnóstico (18 semanas), está aguardando la maduración pulmonar fetal para practicar cesárea e iniciar este esquema.

\section{Conclusión}

La información obtenida en nuestra casuística concuerda con la literatura médica revisada. El CIM continua siendo la forma más agresiva de manifestación del carcinoma mamario.
Estudios más detallados a nivel bioquímico, como la dosificación de receptores de progesterona, de receptores nucleares de estrógeno y el estudio de marcadores tumorales posibilitarían una mejor indicación terapéutica y seguimiento de esas pacientes.

Estudios rutinarios de cinética celular, determinando la tasa de crecimiento tumoral, asume hoy en día un papel preponderante en el tratamiento y conducta posterior de esta enfermedad (26).

En el "Instituto Nazionale per lo studio e La Cura dei Tumori" en Milan, Italia, el tratamiento de los CIM consiste inicialmente en la aspiración de $1000 \mathrm{ml}$ de médula a nivel de la crista ilíaca y quimioterapia en altas dosis con restitución medular posterior cuando las condiciones del paciente lo exijan (Hipoplasia medular) (26).

En la práctica de esas aspiraciones medulares se identifican células neoplásticas a nivel de médula ósea cuando no había sospecha clínica de metástasis. Basados en estos datos, varios investigadores del "Instituto Nazionale per lo studio e la cura dei Tumori" están investigando el uso terapéutico de Anticuerpos Monoclonales marcados con Irídium, I 131, Tecnesio u otras sustancias citotóxicas, en estos tumores (26).

Sin embargo, la posición actual es totalmente contraria a la mastectomía como terapia inicial en el CIM.

Es necesario enfatizar sobre el Carcinoma Inflamatorio de la Mama como entidad clínica definida, de mal pronóstico, punto que siempre debe estar en el pensamiento del médico ante un cuadro de mastitis. Confundir mastitis con carcinoma inflamatorio no amenaza la vida de la paciente, pero diagnosticar y tratar carcinoma inflamatorio como mastitis es muy grave y puede empeorar aún más el pronóstico.

\section{BIBLIOGRAFIA}

1. Bell C. A system of operative surgery vol. 2. Hale and Hosmer, Hardford 1814 , p. 36 "in": Balogh, A, e col. Primary Inflammatory Carcinoma of the breast. Acta Chirurgica Hungarica, 1983; 24 (1)43-57.

2. Volkmann R. Beitrage Zur Chirurgie. Breitkopf and Hartel, Leipziz 1875, pp. 319-334 "in": Balogh, A. e col.: Primary Inflammatory Carcinoma of the breast. Acta Chirurgica Hungarica, 1983; 24 (1): $43-$ 52.

3. Lee BI., Tannenbaum NE. Inflammatory Carcinoma of the breast-Surg. Gynecol. 1924; 39: 580.

4. Haagensen CD. Diseases of the breast. Philadelphia, WB Saunders, 1971.

5. Ellis, David L., Teltebaum, Steve L. Inflammatory Carcinoma of the breast: a pathologic definition. Cancer 1974; 33: 1045-1047.

6. Oliveira LJ., Urbanetz AA. Carcinoma Inflamatório da Mama: Femina, Janeiro 1987; 18-22.

7. Silva Neto, João Baptista. Carcinoma Inflamatório da Mama. A.M.B. V. $13 \mathrm{~N}^{\circ} 8$ 1967; 379-382.

8. Lucas FV., Perez-Mesa C. Inflammatory Carcinoma of the breast. Cancer 1978; 41 : 1595.

9. Saltzstein, Sidney L. Clinically Occult Inflammatory Carcinoma of the breast Cancer 1974; 34: 382-388.

10. Anderson, JM. Inflammatory Carcinoma of the breast. Ann. R. Coll. Surg. Eng. 1980; 62: 195.

11. Robbins GF, Shan J, Rosen P. ChuF., Taylor J. Inflammatory Carcinoma of the breast. Surg. Clin. North Am. 1974; 54: 801-810.

12. Balogh A, y col. Primary Inflammatory Carcinoma of the breast. Act. Chir. Hung. 1983; 24 (1): 43-52.

13. Stocks LH., Patterson FM. Inflammatory Carcinoma of the breast. Surg. Gynecol. Obstet. 1989; 143-6, 885.

14. Pinotti JA. y col. Diagnóstico em Mastologia, Editora Manole Ltda. 1980.
15. Popeu DL y col. Citopunção mamaria por capilaridade: Técnica e análise da eficácia do método 1988. (Trabalho realizado na Unidade de Mastologia do Hospital de Base do DF).(Dados a publicar).

16. Pompeu DL. y col. Forage em Patologia Mamaria, 1988. (Trabalho a publicar).

17. Zylberberg B. y col. Initial Chemoimmuno therapy in Inflammatory Carcinoma of the breast. Cancer 1982; 49: 1537-1543.

18. Wang CC. Management of Inflammatory Carcinoma of the breast. JAMA 1967; 201-123.

19. Delarue JC. y col. Oestrogen and progesterone cytosolic receptors in clinically Inflammatory tomours of the human breast. Br. J. Cancer 1981; 44: 911.

20. Pinotti JA. y col. Terapêutica em Mastologia Editora Manole Ltda. 1982; 245-249.

21. Camp E. Inflammatory Carcinoma of the breast, the case for conservotism The American Journal of Surgery 1976; 131: 583-586.

22. Chu, AM., Wood W., Doucette J. Inflammatory breast Carcinoma treated by Radical Radio Therapy. Cancer 1980; 45: 2730-2737.

23. Buzdar A. y col. Management of Inflammatory Carcinoma of Breast With Combined Modality App ROA cth - An Update. Cancer 1981; 47 : 2537-2542.

24. Rouessé J. y col. Chimiotherapie premiere dans le traitement des cancers du sein Inflammatoires. Presse Méd. 16: 475-478.

25. Da Luz, Doris A. Unidade de Radioterapia - Hospital do Base do Distrito Federal - Comunicação Pessoal.

26. Lima LRS. Mastologista do Hospital de Base do Distrito Federal. Comunicação Pessoal de seu estágiao no "Instituto Nazionale Per Lo Studio e La Cura Dei Tumori” durante o período de Setembro de 1987 a Fevreiro de 1988. 